\title{
Investigation on Optical Properties of Natural Brown Diamonds with Various Types by High Pressure and High Temperature Treatment
}

\author{
Jong-Hyuck Bai****, Jin-Gyo Seo***, Shoo-Hack Shon*, \\ Yong-Kil Ahn*** and Jong-Wan Park**** \\ *Department of Materials and Chemical Engineering, Graduate School of Engineering, \\ 17 Haengdang-dong, Seongdong-gu, Hanyang University, Seoul 133-791, Korea \\ **Central Gemological Institute, Busan 834-4, Korea \\ ***:Department of Materials Science and Engineering, Hanyang University, 17 Haengdang-dong, \\ Seongdong-gu, Seoul, 133-791, Korea
}

(2010년 5월 11일 접수 : 2010년 5월 17일 최종수정 : 2010년 5월 18일 채택)

\begin{abstract}
High Pressure High Temperature (HPHT) treatment can significantly change the color of diamonds. We studied the variation of the optical properties according to the nitrogen arrangement in natural brown diamonds of various types (type IaAB, type IaB, type IaA $>$ B, type IaA $<$ B, IaA $=\mathrm{B}$ ) after HPHT treatment. The diamonds with different arrangements of nitrogen were annealed at temperatures in the range $1700-1800^{\circ} \mathrm{C}$ under a stabilizing pressure of $5 \mathrm{GPa}$. HPHT treated samples were analyzed using UV-Vis-NIR, FT-IR, and PL spectroscopy. The absorption and luminescence spectra were measured to compare the variations of nitrogen arrangement in the natural brown diamonds before and after HPHT treatment. After HPHT treatment, the brown coloration in all types of diamonds was reduced and a decrease in the peaks related to the A-aggregate of nitrogen was more predominant than the B-aggregate. Furthermore, the peaks related to N3 $(415.4 \mathrm{~nm}), \mathrm{H} 4(496.4 \mathrm{~nm})$, and platelet decreased and the peaks related to $\mathrm{H} 3(503.2 \mathrm{~nm})$ and G-band increased after HPHT treatment. In conclusion, spectroscopic analysis of natural brown diamonds after HPHT treatment showed that a yellow color was produced by absorption in the H3 centers and a green color was generated by interaction between absorptions of the $\mathrm{H} 3$ and $\mathrm{H} 2$ centers.
\end{abstract}

Key words brown diamond, HPHT treatment, optical properties characterization, defects.

\section{1. 서 론}

다이아몬드는 탄소(carbon)로 이루어진 무기질 광물로 보석용 다이아몬드는 결정이 생성될 당시의 환경 등에 의 해 아주 미량의 불순물들(impurities)이 포함될 수 있다. 이러한 미량의 불순물들은 결정의 색이나 구조에 영향을 미치며, 불순물의 유무, 종류, 결합형태에 따라 다이아몬 드의 type이 결정된다. 특히, 다이아몬드는 불순물로 대 다수 질소(nitrogen)를 포함하기 때문에 다이아몬드의 type 을 구분함에 있어 질소의 결합상태는 상당히 중요하다.

다이아몬드는 질소의 존재 유무에 따라 type I (질소가 약10 5,500 ppm 불순물로 존재)과 type II (질소가 10 $\mathrm{ppm}$ 이하로 거의 없는 경우)로 나눌 수 있다. Type I 에 속한 다이아몬드는 질소원자가 집합체로 존재하는 type $\mathrm{Ia}$, 질소원자가 고립되어 단독으로 존재하는 type $\mathrm{Ib}$ 로 나 뉘며, type Ia는 세부적으로 질소의 배열상태에 따라 $\mathrm{IaA}$, $\mathrm{IaB}, \mathrm{IaAB}$ 로 구분된다. ${ }^{1-3)}$ Type $\mathrm{II}$ 의 경우, 질소가 거의

'Corresponding author

E-Mail : jwpark@hanyang.ac.kr (J. -W. Park)
없는 type IIa, 붕소(boron)원자들이 불순물로 존재하는 type IIb로 나누어 진다. 일반적으로 다이아몬드의 색은 질소의 함량에 따라 황색을, 붕소의 함량에 따라 청색 을 띈다. 다이아몬드의 brown color는 type IIa의 경우, 소성변형(plastic deformation)에 의해 발생된다고 잘 알 려져 있다. ${ }^{4-5)}$ 따라서 고온고압처리(High Pressure High Temperature treatment; HPHT)를 통해 소성변형을 치유 하여 다이아몬드의 색을 없앨 수 있는 것으로 알려져 있 다. ${ }^{6)}$ 하지만 type $\mathrm{Ia}$ 의 경우, brown color의 원인은 소성 변형 외에도 다른 여러 가지 원인들에 의해 발생될 수 있 다. Type Ia brown 다이아몬드의 경우 그 color는 amber center와도 연관이 있다고 잘 알려져 있다. ${ }^{7-10)}$ Type Ia brown 다이아몬드는 HPHT 처리 시 type IIa와는 달리 소성변형의 치유 외에도 다양한 광학적 변화가 나타날 수 있다.

따라서 본 연구에서는 HPHT 처리 전 FT-IR spectrometer를 이용한 사전분석을 통해 type Ia brown 다이아 몬드를 $\mathrm{IaA}, \mathrm{IaB}, \mathrm{IaAB}(\mathrm{A}>\mathrm{B}), \mathrm{IaAB}(\mathrm{A}=\mathrm{B}), \mathrm{IaAB}$ $(\mathrm{A}<\mathrm{B})$ 로 세부적으로 분류하고, amber center의 유무 및 $\mathrm{HPHT}$ 처리 후 질소결합상태의 변화 등을 비교 관찰하 
여 color와의 상관관계에 대해 규명하고자 하였다.

\section{2. 실험 방법}

본 연구에서는 FT-IR spectrometer 사전 분석을 통해 다 이아몬드 내 불순물로 존재하는 질소의 결합상태에 따라 $\mathrm{IaA}, \mathrm{IaB}, \mathrm{IaAB}(\mathrm{A}>\mathrm{B}), \mathrm{IaAB}(\mathrm{A}=\mathrm{B}), \mathrm{IaAB}(\mathrm{A}<\mathrm{B})$ 으로 분류하여 선택된 총 5 개의 type Ia brown 다이아 몬드 (0.198 0.719 ct)를 사용하였다. 보석학적 기초검사를 위해 MARK VII 보석용 쌍안현미경으로 HPHT 처리 전 과 후의 외관검사 및 내포물 검사를 실시 하였다. HPHT 처리는 $1700-1800^{\circ} \mathrm{C}, 5 \mathrm{GPa}$ 에서 다이아몬드가 흑연화 되 지 않는 범위 하에서 실시하였다. 시료의 분광학적 특 성은 자외선-가시광선 분광분석기(UV-Vis Spectrometer, Shimadzu - UV 3101PC)를 사용하여 350 800 nm에서의 가시광선 범위를 $0.1 \mathrm{~nm}$ 의 분해능으로 투과(Transmittance) 모드로 측정하였고, 퓨리에 변환 적외선 분광분석기(FTIR spectrometer, Jasco-4100)을 사용하여 $400 \sim 6000 \mathrm{~cm}^{-1}$ 의 범위에서 $4 \mathrm{~cm}^{-1}$ 의 분해능으로 흡수(Absorption) 모드 로 측정한 후 HPHT 처리 전후를 비교 분석하였다. 또한 광루미네선스(Photoluminescence) 분석은 $325 \mathrm{~nm} \mathrm{He-Cd}$ laser를 광원으로 한(PL, Spectra-pro 2150i, Spectra-pro $2300 \mathrm{i}$ micro-spectrometer) 및 $532 \mathrm{~nm}$ green laser를 광 원으로 한(PL, SAS 2000)를 사용하여 각각 $350 \sim 600 \mathrm{~nm}$, $550 \sim 1100 \mathrm{~nm}$ 의 범위에서 $0.1 \mathrm{~nm}$ step으로 측정하여 HPHT 처리 전과 후를 비교 분석하였다.

\section{3. 결과 및 고찰}

\section{1 보석학적 기초검사}

\subsection{1 형광성 검사}

Table 1은 HPHT 처리 전 후의 형광 검사결과를 나타 내는 표이다. HPHT 처리 전 모든 시료는 blue형광을 나 타내었고, 처리 후에는 yellowish green의 형광성을 보였 다. 처리 전과 후 형광성의 정도는 Table 1 에서 보는 바 와 같이 특정한 경향성을 나타내진 않았다. 하지만 HPHT 처리 후 다이아몬드 실체색(body color)는 다이아몬드 내 불순물인 질소를 $\mathrm{A}$ 집합으로 많이 포함하고 있을 경우 greenish yellow으로, B 집합이 많은 경우 yellowish green 를 나타내는 경향을 보였다.

\section{1 .2 내포물 검사}

Fig. 1은 HPHT 처리 전과 후 내포물의 변화를 관찰한 결과이다. 실험에 사용된 총 5 개의 시료 중 2 개의 시료 ((a) type $\mathrm{IaB}$, (c) type $\mathrm{IaA})$ 는 HPHT처리 전 각각 흑점 (black spot)과 다이아몬드 내부에 제한되어 있는 패더 (feather)를 동반한 내포물을 가진 다이아몬드였다. 두 시 료는 Fig. 1에서 보는 바와 같이 HPHT 처리 전 (a) type $\mathrm{IaB}$ 시료의 black spot(표시된 부분)이 HPHT 처리 후 (b) 모두 사라졌고, (c) type IaA시료의 경우, HPHT처리 전에 내부에만 존재하던 feather(표시된 부분)가 HPHT처리 후(d) 외부까지 확장됨과 동시에 흑연화된 패더(graphitized feather)의 특징을 나타내었다.

\subsubsection{Strain pattern검사}

Fig. 2는 교차편광필터(closed polarizing filter)를 이용 하여 다이아몬드의 HPHT처리 전과 후의 strain pattern 을 관찰한 결과를 나타내었다. 본 연구에 사용된 모든 시 료는 type Ia의 범주에 속해 있으므로 type Ia에서 나타 나는 전형적인 무지개 타입의 strain pattern을 관찰할 수 있었다. Fig. 2에서 보는 바와 같이 HPHT 처리 전(a)과 후(b) strain pattern의 변화는 나타나지 않았다.

\section{2 퓨리에 변환 적외선(FT-IR) 분광분석 결과}

Fig. 3은 brown 다이아몬드의 HPHT 처리 전과 후의 퓨리에 변환 적외선 분광분석(FT-IR) 측정 결과이다. 일 반적으로 다이아몬드 시료는 $400 \sim 6000 \mathrm{~cm}^{-1}$ 의 영역에서 네 구간의 phonon 영역이 있다. 그 중 one- phonon region $\left(1332 \mathrm{~cm}^{-1}\right.$ 이하의 영역)이라 불리는 $400 \sim 1500 \mathrm{~cm}^{-1}$ 은 질소와 연관된 흡수 진동이 있는 곳이다. ${ }^{11)}$ 따라서 이 영역의 흡수 유무에 따라 다이아몬드를 type I과 type II

Table 1. Fluorescence inspection of diamond before and after HPHT treatment.

(a) Before HPHT Treatment

\begin{tabular}{|c|c|c|c|}
\hline $\begin{array}{l}\text { Sample } \\
\text { No. }\end{array}$ & Sample Type & $\begin{array}{l}\text { Short Wave } \\
\text { (SW) }\end{array}$ & $\begin{array}{l}\text { Long Wave } \\
\text { (LW) }\end{array}$ \\
\hline & & \multicolumn{2}{|c|}{ Fluorescence color : Blue } \\
\hline 501 & Type IaB & None & Mediun \\
\hline 502 & Type IaAB $(\mathrm{A}>\mathrm{B})$ & None & None \\
\hline 503 & Type IaA & None & None \\
\hline 506 & Type IaAB $(A=B)$ & None & None \\
\hline 513 & Type IaAB $(\mathrm{A}<\mathrm{B})$ & None & Week \\
\hline
\end{tabular}

(b) After HPHT Treatment

\begin{tabular}{cccc}
\hline $\begin{array}{c}\text { Sample } \\
\text { No. }\end{array}$ & Sample Type & $\begin{array}{c}\text { Short Wave } \\
(\mathrm{SW})\end{array}$ & $\begin{array}{c}\text { Long Wave } \\
(\mathrm{LW})\end{array}$ \\
\hline & & Fluorescence color : Yellowish Green \\
501 & Type IaB & Week & Medium \\
502 & Type IaAB $(\mathrm{A}>\mathrm{B})$ & Week & Medium \\
503 & Type IaA & Medium & Strong \\
506 & Type IaAB $(\mathrm{A}=\mathrm{B})$ & Medium & Strong \\
513 & Type IaAB $(\mathrm{A}<\mathrm{B})$ & Medium & Strong \\
\hline
\end{tabular}



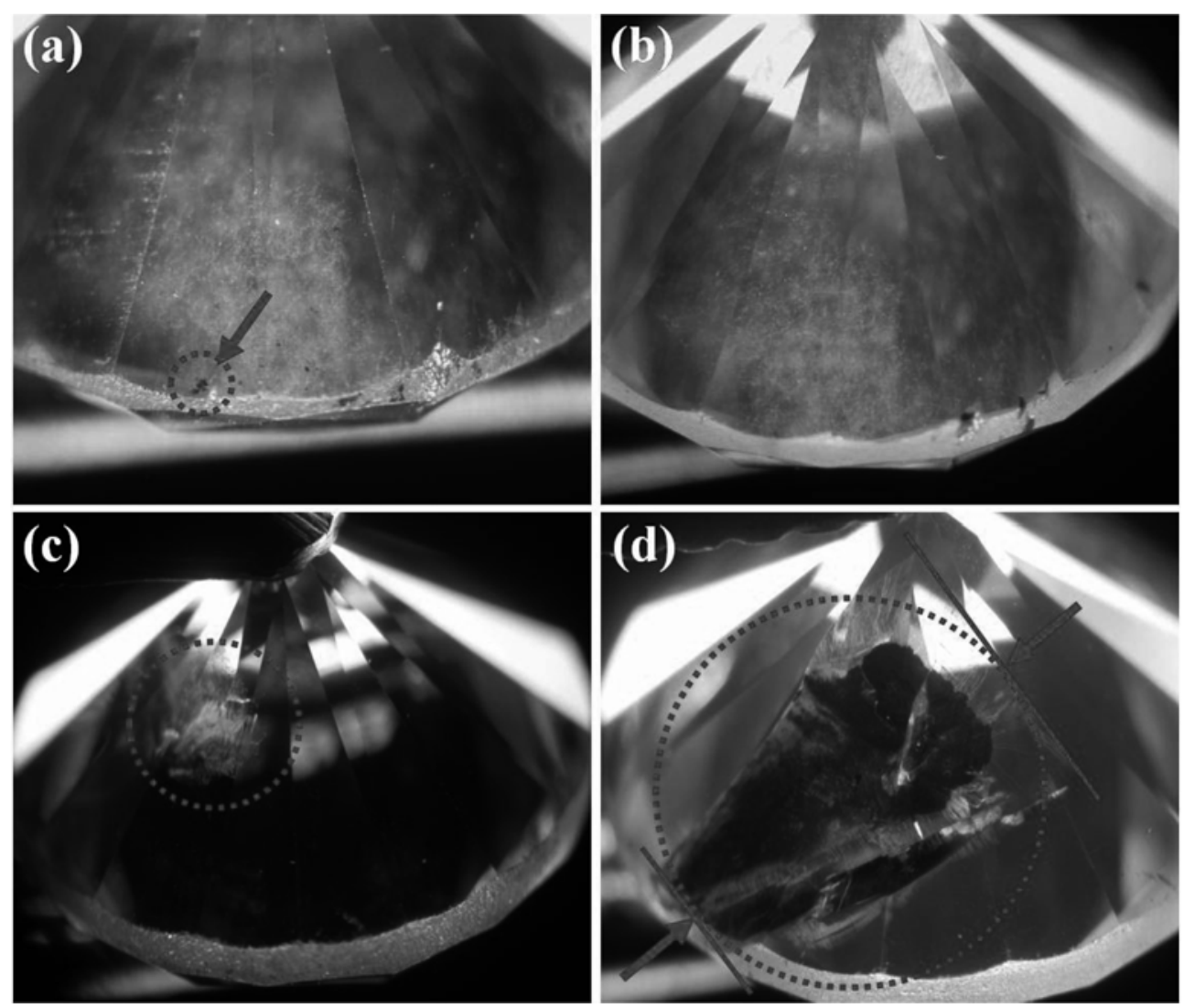

Fig. 1. Variation of inclusion in diamond before (a, c) and after (b, d) HPHT treatment; (a-b) type IaB (sample no.501) and (c-d) type IaA (sample no. 503).
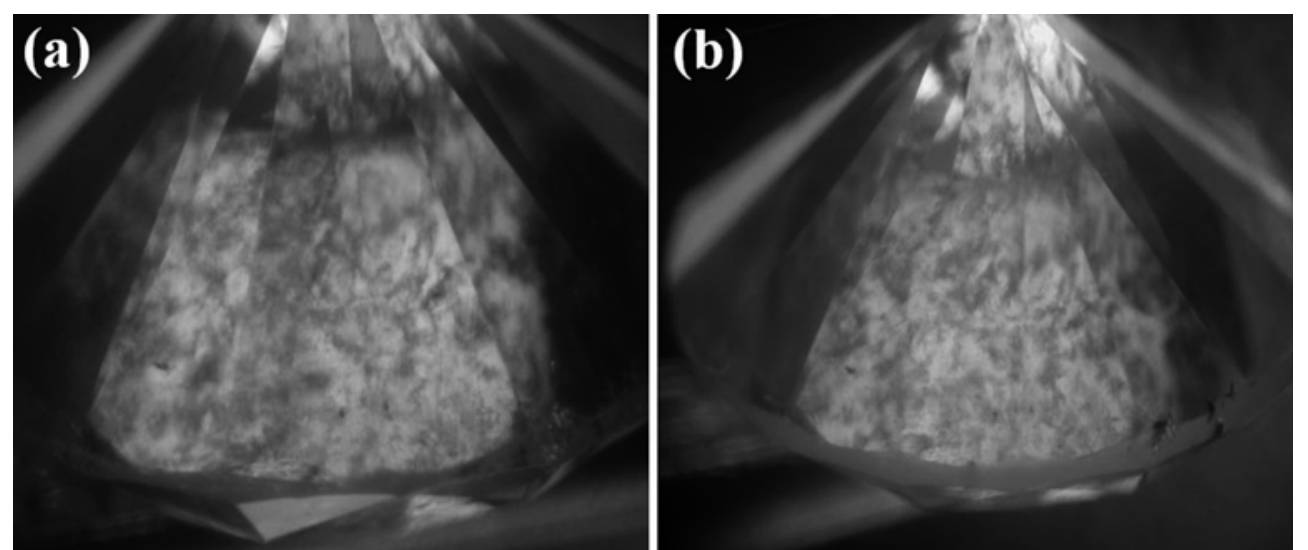

Fig. 2. Strain pattern variation of diamond before and after HPHT treatment (sample no. 506); (a) before HPHT treatment and (b) After HPHT treatment.

로 구분할 수 있으며, 질소의 흡수 패턴에 따라 세부적인 type 구분이 가능하다. 또한 three-phonon region $\left(3994 \mathrm{~cm}^{-1}\right.$ 이하의 영역)은 수소(hydrogen)와 연관된 흡수 진동이 있 는 곳이며, 수소와 연관된 흡수 진동 peak의 유무에 따 라 천연과 합성을 감별하기도 한다. Four-phonon region $\left(5328 \mathrm{~cm}^{-1}\right.$ 이하의 영역)은 amber center $\left(4160 \mathrm{~cm}^{-1} \pm 100\right)$ 와 관련이 있는 흡수 진동이 있는 곳으로 잘 알려져 있 다. ${ }^{12)}$ 따라서 우리는 각 시료에 대한 정보를 구체적으로
확인하기 위해 세 영역으로 나누어 확대 한 스펙트럼으 로 (a-1 e-3) HPHT 처리 전과 후를 비교 관찰하였다. 여 기에서 two-phonon region $\left(2664 \mathrm{~cm}^{-1}\right.$ 이하의 영역 $)$ 은 다 이아몬드의 진성 결합(intrinsic band) 영역으로 별 특이한 흡수 진동이 없으므로 제외시켰다. Fig. 3에서 보는 바와 같이 5 개의 시료는 모두 질소를 불순물로 포함하는 type $\mathrm{I}$ 이며 (a)는 type IaB, (b)는 type $\mathrm{IaAB}(\mathrm{A}>\mathrm{B})$, (c)는 type $\mathrm{IaA},(\mathrm{d})$ 는 type $\operatorname{IaAB}(\mathrm{A}=\mathrm{B}),(\mathrm{e})$ 는 type $\operatorname{IaAB}(\mathrm{A}<\mathrm{B})$ 

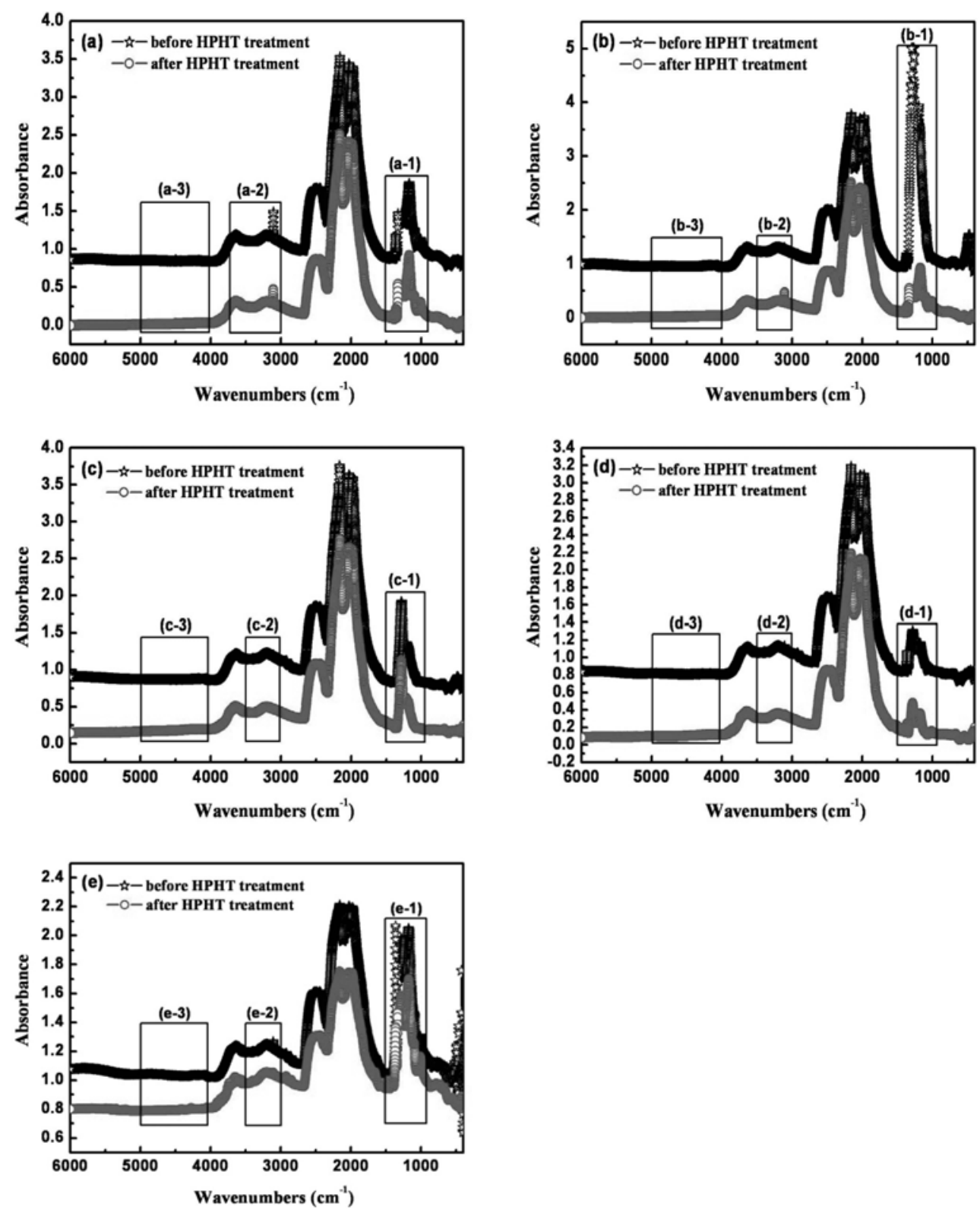

Fig. 3. Comparison of the FT-IR spectra with various types of diamonds before (black line) and after (red line) HPHT treatment; (a) type $\mathrm{IaB}$, (b) type $\mathrm{IaAB}(\mathrm{A}>\mathrm{B})$, (c) type $\mathrm{IaA}$, (d) type $\mathrm{IaAB}(\mathrm{A}=\mathrm{B})$ and $(\mathrm{e})$ type $\mathrm{IaAB}(\mathrm{A}<\mathrm{B})$.

임을 알 수가 있다. 시료 (a)의 경우 아주 미량의 $\mathrm{A}$ 집 합 $\left(1282 \mathrm{~cm}^{-1}\right)$ 이 존재하기는 하나 type $\mathrm{IaB}$ 에 가까운 시 료라 할 수 있다.

Fig. 4는 각 시료의 질소와 연관된 흡수진동이 있는 곳 을 나타낸 결과이다. Fig. 4에서 보는 바와 같이 HPHT 처 리 후 대부분의 흡수는 전체적으로 감소하는 경향을 나 타내었다. 특히 (b-1)의 type $\mathrm{IaAB}(\mathrm{A}>\mathrm{B})$ 시료는 $\mathrm{A}$ 집합이 확연히 감소하는 것을 확인할 수 있었다. 또한 $1363 \mathrm{~cm}^{-1}$ 의 platelet과 연관된 peak가 HPHT처리 후 모든 시료에 서 감소한 것을 확인할 수 있었다. $1010 \mathrm{~cm}^{-1}$ 와 $1405 \mathrm{~cm}^{-1}$ 는 $3107 \mathrm{~cm}^{-1}$ peak와 항상 함께 나타나는 것을 알 수 있
다. 이는 $1010 \mathrm{~cm}^{-1}$ 및 $1405 \mathrm{~cm}^{-1}$ 의 peak는 수소와 연관된 peak임을 알 수 있으며, Fig. 5. (c-2)에서 확인할 수 있는 $3107 \mathrm{~cm}^{-1}$ 의 부재로 인해 (c-1)의 spectrum에서 $1010 \mathrm{~cm}^{-1}$ 및 $1405 \mathrm{~cm}^{-1}$ 의 peak는 나타나지 않았다. $1095 \mathrm{~cm}^{-1}$ 의 peak 는 주로 질소를 불순물로 포함 (A 또는 B집합)하고 있는 type I에서 주로 나타나는 peak이다. ${ }^{13)}$ (d-1) 스펙트럼에 서 나타난 $1215 \mathrm{~cm}^{-1}$ 의 peak는 질소의 A집합 one-phonon 흡수 특징을 나타낸다.

Fig. 5는 three-phonon 영역의 흡수 진동을 나타낸다. 수소와 연관된 $3107 \mathrm{~cm}^{-1}$ 의 흡수 진동 peak는 HPHT 처 리 후 대다수 감소하는 경향을 보였으나, (b-2)의 type 

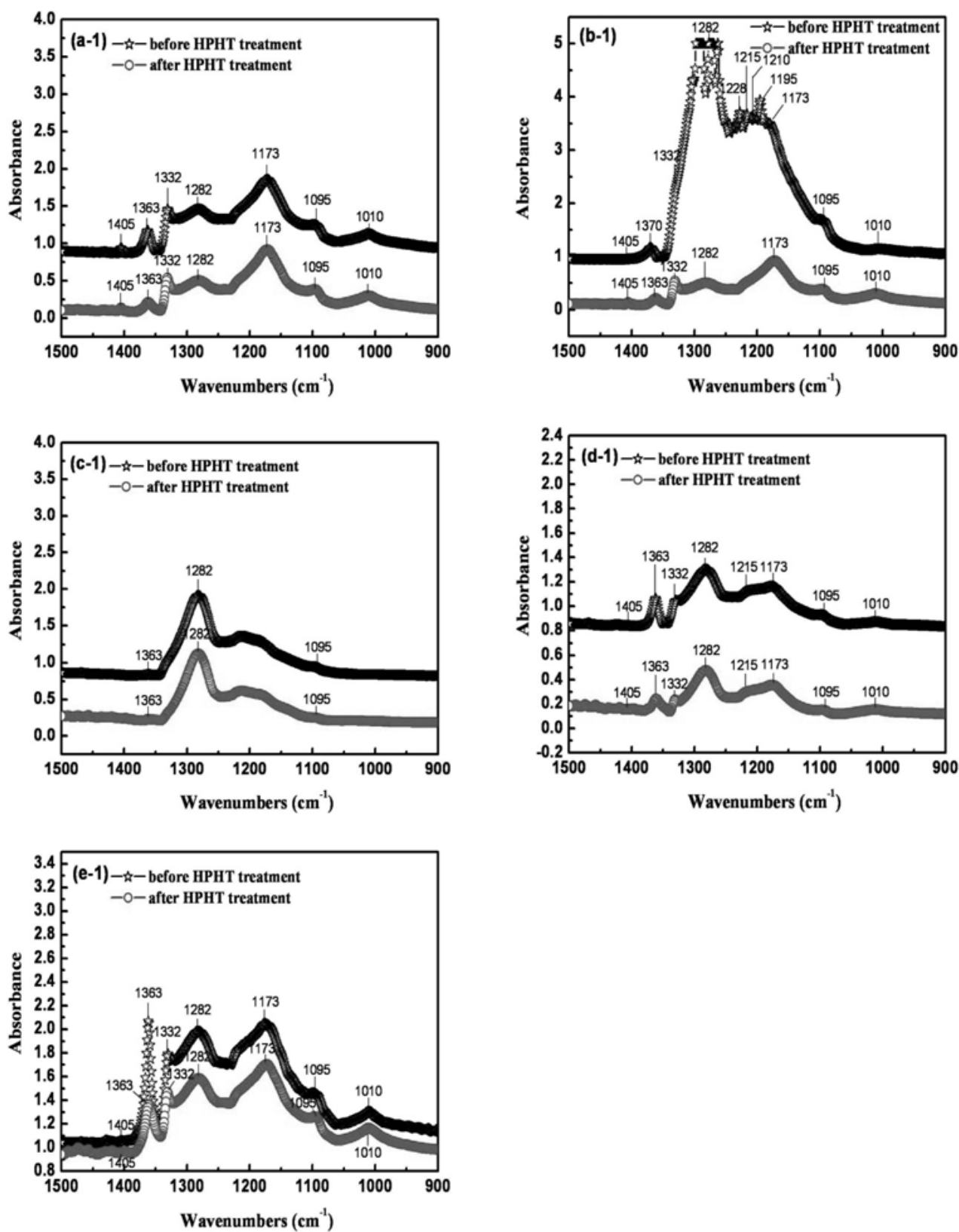

Fig. 4. FT-IR absorption enlarged spectra of $900 \mathrm{~cm}^{-1} \sim 1500 \mathrm{~cm}^{-1}$ in one-phonon region (a-1 e-1) before (black line) and after (red line) HPHT treatment; (a-1) type IaB, (b-1) type IaAB (A > B), (c-1) type IaA, (d-1) type IaAB $(\mathrm{A}=\mathrm{B})$ and $(\mathrm{e}-1)$ type IaAB $(\mathrm{A}<\mathrm{B})$.

$\operatorname{IaAB}(\mathrm{A}>\mathrm{B})$ 시료에서는 오히려 증가하는 결과를 나타내 었다. $3107 \mathrm{~cm}^{-1}$ 의 수소관련 peak는 HPHT 처리 후에도 대다수 시료에서 존재함을 확인할 수 있었다.

Fig. 6는 four-phonon 영역의 흡수 진동을 관찰한 결 과이다. Type Ia brown 다이아몬드는 $4160 \mathrm{~cm}^{-1} \pm 100$ 에 서 amber center와 연관된 peak가 나타나는 것으로 알려 져 있으나, ${ }^{12}$ Fig. 6에서 보는 바와 같이 본 실험에 사용 한 모든 시료 (a-3 e-3)에서는 amber center와 연관된 흡 수 진동 peak를 확인할 수 없었다. 이러한 결과는 type Ia brown 다이아몬드에서 amber center가 항상 존재하는
것이 아님을 나타내며, 본 실험에 사용된 다이아몬드의 경 우, brown color는 amber center 외 다른 이유로 brown color가 발색된 것으로 추정된다. 기존의 보고에 따르면 brown color는 다이아몬드 격자 내 존재하는 공공(vacancy), 변형(strain), 전위(dislocation) 또는 질소 불순물들에 의해 생성된 많은 platelets과 다양한 color center들과의 상호 작용을 통해 발색될 것이라 추측될 뿐, 실제로 type Ia 다이아몬드에 있어 brown color의 발색 기원을 명확히 보고한 사례는 없다. 다만 amber center의 경우, type II 다이아몬드에는 나타나지 않고, 대다수 type Ia brown 다 

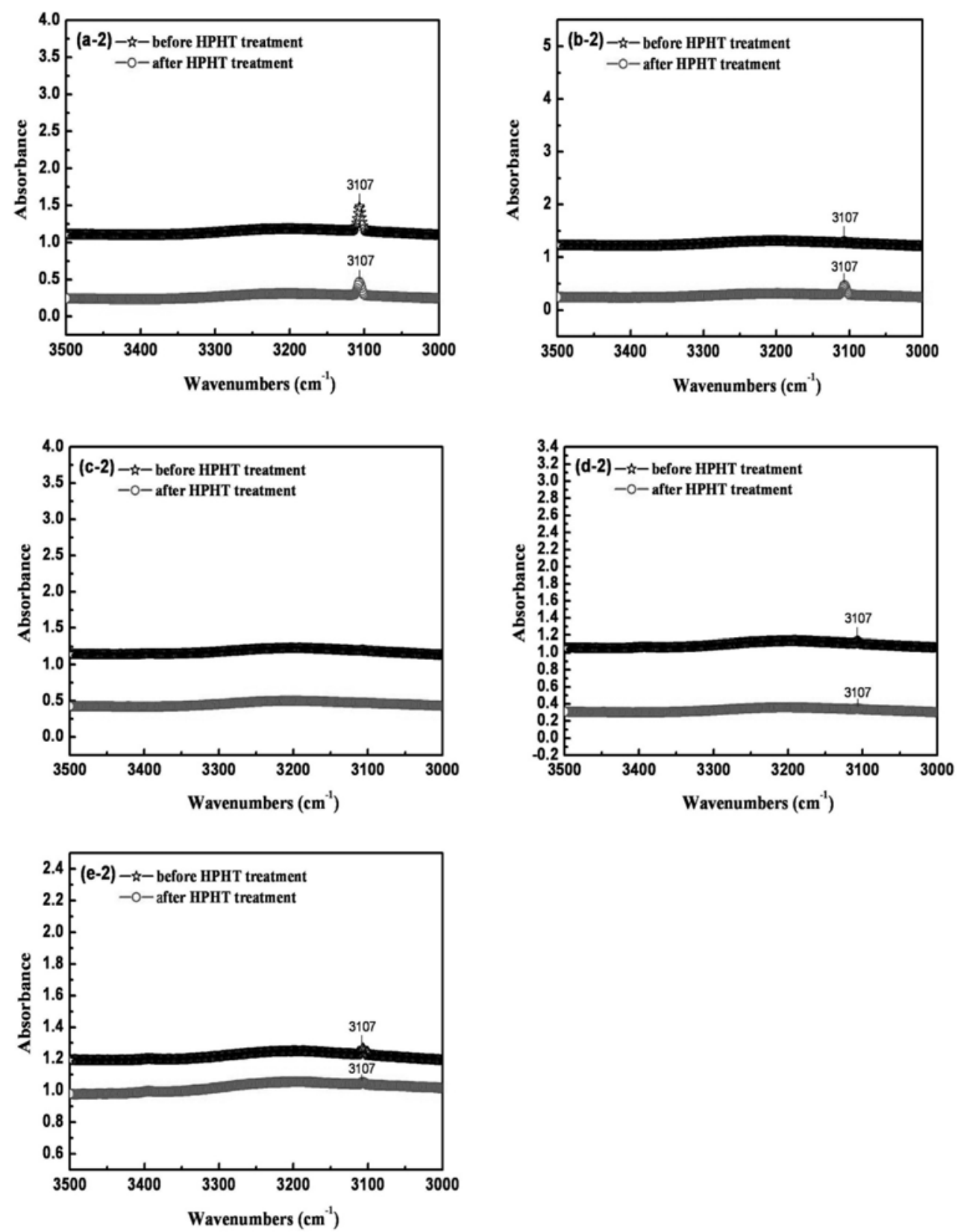

Fig. 5. FT-IR absorption enlarged spectra of $3000 \mathrm{~cm}^{-1} \sim 3500 \mathrm{~cm}^{-1}$ in two-phonon region (a-2 e-2) before (black line) and after (red line) HPHT treatment; (a-2) type IaB, (b-2) type IaAB $(\mathrm{A}>\mathrm{B}),(\mathrm{c}-2)$ type IaA, $(\mathrm{d}-2)$ type $\mathrm{IaAB}(\mathrm{A}=\mathrm{B})$ and $(\mathrm{e}-2)$ type IaAB $(\mathrm{A}<\mathrm{B})$.

이아몬드에 존재 (일부 type $\mathrm{Ib}$ 의 경우에 나타나기도 함) 하기 때문에 type Ia brown 다이아몬드의 경우, amber center 가 brown color 발색에 있어 일부 영향을 줄 것이 라 보고 된 바 있다. ${ }^{12)}$ 특이하게 (a-3)시료의 경우, HPHT 처리 전 $4498 \mathrm{~cm}^{-1}$ 에서 흡수 진동 peak가 나타나는데 이는 $3107 \mathrm{~cm}^{-1}$ 에서 나타나는 peak와 마찬가지로 수소와 연관된 peak이며, HPHT처리 후에는 사라짐을 확인할 수 있었다.

\section{3 자외선-가시광선(UV-Vis) 분광 분석결과}

Fig. 7는 HPHT 처리 전 후의 자외선-가시광선 분광
분석(UV-Vis spectrometer) 결과를 나타낸다. Fig. 7에서 보는 바와 같이 HPHT 처리 후 모든 시료는 $503 \mathrm{~nm}$ 의 중성 전하를 띈 $\mathrm{H} 3(\mathrm{~N}-\mathrm{V}-\mathrm{N})^{0}$ 의 흡수가 발생함과 동시에 G-band가 생성됨을 확인할 수 있다. 모든 시료는 HPHT 처리 후 G-band 생성으로 인해 다이아몬드의 color가 brown에서 yellowish green으로 바뀌는 것을 알 수 있 다. 그러나 본 연구에서는 $800 \mathrm{~nm}$ 이상에서 심한 noise 로 인해 $986 \mathrm{~nm}$ 의 $\mathrm{H} 2$ center $(\mathrm{N}-\mathrm{V}-\mathrm{N})^{-}$를 확인하지 못하 였지만, $\mathrm{H} 2$ center의 생성이 $\mathrm{H} 3$ center 및 $\mathrm{G}$ band와 상 호 작용하여 다이아몬드의 color를 yellowish green하게 

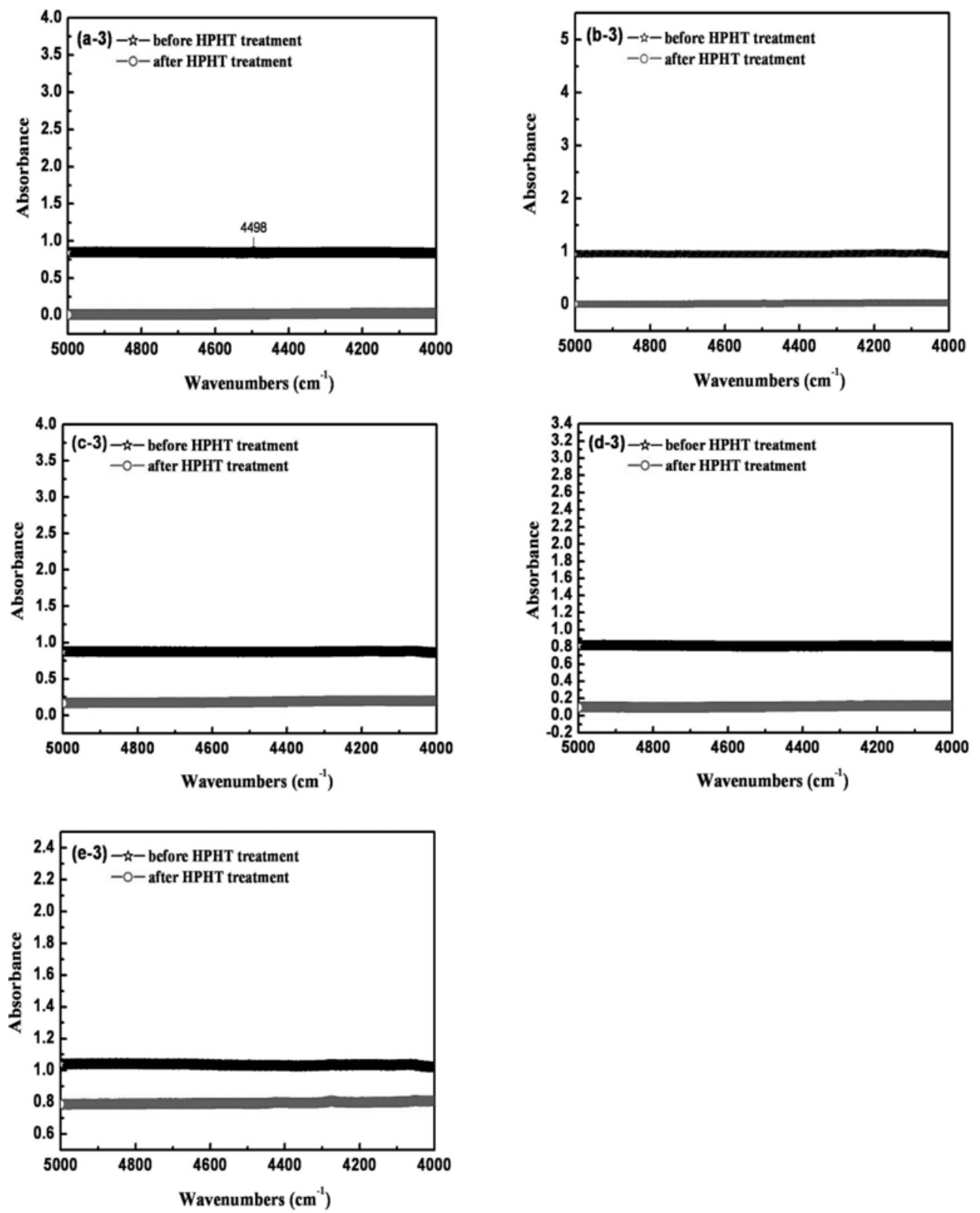

Fig. 6. FT-IR absorption enlarged spectra of $4000 \mathrm{~cm}^{-1} \sim 5000 \mathrm{~cm}^{-1}$ in four-phonon region (a-3 e-3) before (black line) and after (red line) HPHT treatment; (a-3) type IaB, (b-3) type IaAB $(\mathrm{A}>\mathrm{B}),(\mathrm{c}-3)$ type $\mathrm{IaA},(\mathrm{d}-3)$ type $\mathrm{IaAB}(\mathrm{A}=\mathrm{B})$ and $(\mathrm{e}-3)$ type IaAB $(\mathrm{A}<\mathrm{B})$.

만든 것으로 생각된다. 이러한 color center의 변화를 좀 더 명확히 관찰하기 위해 $325 \mathrm{~nm} \mathrm{He-Cd} \mathrm{laser와} 532 \mathrm{~nm}$ green laser의 두 광원을 사용, 구간별로 $\mathrm{PL}$ 분석을 실 시하였다.

\section{4 광루미네선스 $(\mathrm{PL})$ 분광분석결과 $(325 \mathrm{~nm} \mathrm{He}-\mathrm{Cd}$ laser source)}

Fig. 8은 HPHT 처리 전 후의 $325 \mathrm{~nm} \mathrm{He}-\mathrm{Cd}$ laser를 광 원으로 한 PL분석 결과를 나타낸다. 모든 시료는 HPHT 처 리 후 $\mathrm{N} 3$ center $(415.4 \mathrm{~nm} ; 3 \mathrm{~N}-\mathrm{V})$ 와 이와 연관된 phonon 들 $(428.5,439.6,453.8,463.9 \mathrm{~nm})$ 이 감소하였고, 자외선
-가시광선 분광분석 결과와 마찬가지로 G-band가 생성되 는 것을 확인할 수 있었다. 또한 HPHT 처리 전 모든 시 료에 존재했던 H4 center $(496.4 \mathrm{~nm} ; 4 \mathrm{~N}-2 \mathrm{~V})$ 는 HPHT 처리 후 모두 사라짐을 알 수 있고, G-band의 생성과 함 께 $(\mathrm{N}-\mathrm{V})^{0}$ center $(575.9 \mathrm{~nm})$ 가 증가함을 알 수 있다. H4 center는 불안정한 격자 구조로 인해 HPHT 처리 시 대 다수 파괴된다고 보고된 바 있다. ${ }^{14-15)} \mathrm{Fig}$. 8의 (b) - type $\mathrm{IaAB}(\mathrm{A}>\mathrm{B})$ 와 $(\mathrm{c})$ - type $\mathrm{IaA}$ 의 시료는 $\mathrm{HPHT}$ 처리 전 $398.8 \mathrm{~nm}$ 의 peak가 HPHT 처리 후 감소한 것으로 보아 질소 불순물의 $\mathrm{A}$ 집합과 연관된 peak로 보인다. 이는 적 외선 분광분석 결과에서 나타낸 바와 같이 HPHT 처리 

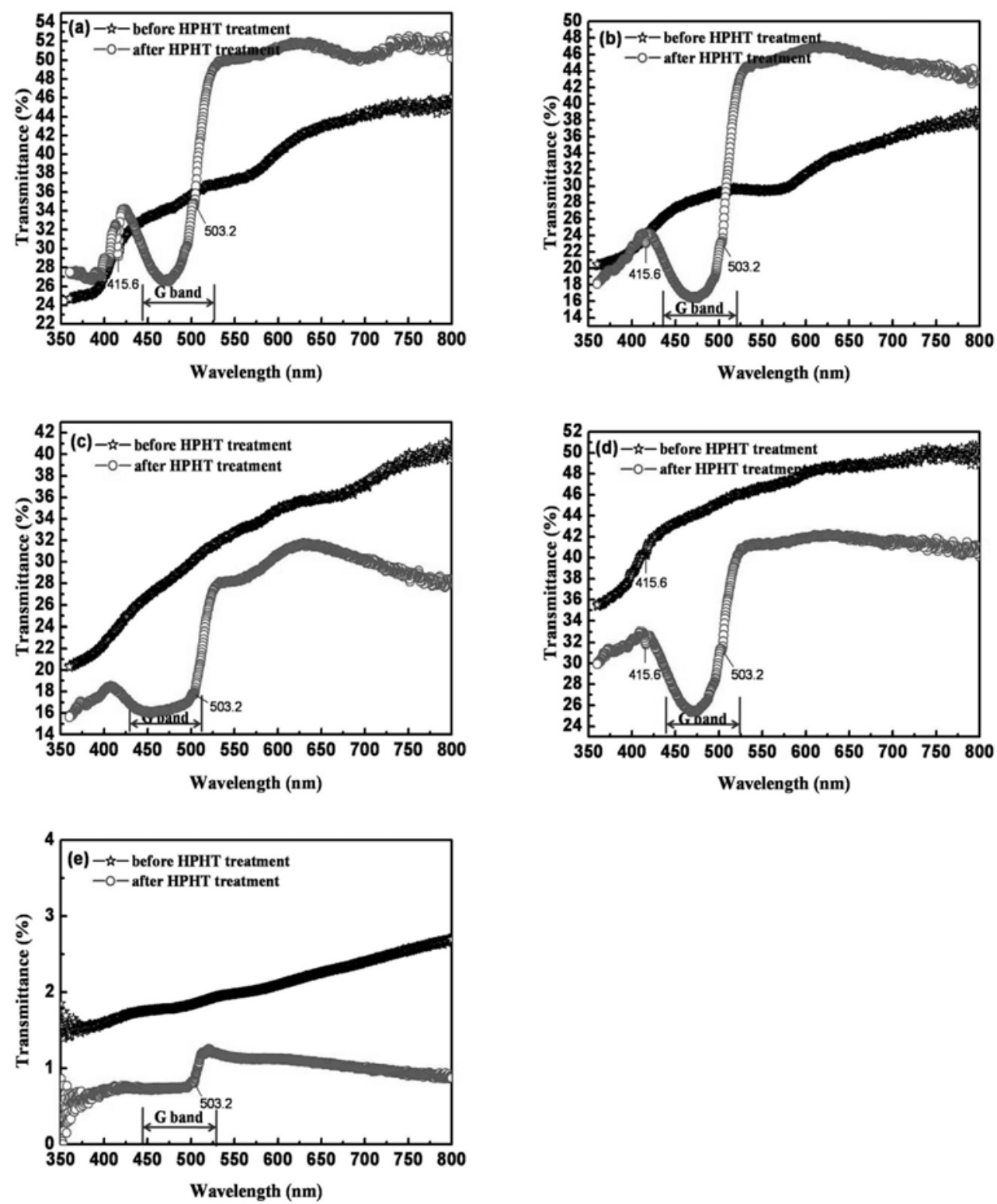

Fig. 7. UV-Vis spectrometer absorption spectra with various types of diamonds before (black line) and after (red line) HPHT treatment; (a) type $\mathrm{IaB}$, (b) type $\mathrm{IaAB}(\mathrm{A}>\mathrm{B})$, (c) type $\mathrm{IaA}$, (d) type $\mathrm{IaAB}(\mathrm{A}=\mathrm{B})$ and (e) type $\mathrm{IaAB}(\mathrm{A}<\mathrm{B})$.

후에는 질소의 $\mathrm{A}$ 와 $\mathrm{B}$ 집합체가 감소하나, $\mathrm{B}$ 집합체보다 $\mathrm{A}$ 집합체가 더 많이 감소한 결과와 일치한다.

\section{4 광루미네선스 $(\mathrm{PL})$ 분광분석결과(532 nm green laser source)}

Fig. 9은 HPHT 처리 전 후의 $532 \mathrm{~nm}$ green laser를 광 원으로 한 PL분석 결과를 나타낸다. $1064 \mathrm{~nm}$ 의 saturation 된 peak는 $532 \mathrm{~nm}$ 의 laser 광원의 second laser peak이 다. HPHT 처리 후 모든 시료에서 HPHT처리 전에 존재 하지 않던 $(\mathrm{N}-\mathrm{V})^{-}$center $(637.4 \mathrm{~nm})$ 가 생성됨을 확인 할 수 있다. 또한 천연 다이아몬드에서 주로 나타나는 $612.4 \mathrm{~nm}$ 의 peak는 모두 사라짐을 알 수 있다. 특이하게도 HPHT
처리 후 모든 시료는 $594 \mathrm{~nm}$ 의 침입형센터(interstitial center)와 연관된 peak가 증가하는 경향을 보였다. 이는 HPHT 처리 시 A center 또는 B center가 파괴되면서 발 생한 질소 원자의 일부가 다이아몬드 격자 내로 interstitial 되면서 나타나는 현상이라 사료된다. 또한 HPHT 처리 전 에는 없었던 $600,620,659.3,681.4 \mathrm{~nm}$ 의 peak가 HPHT 처리 후 새로이 생성됨을 알 수 있었다.

\section{4. 결 론}

본 연구에서 질소의 결합상태에 따라 type Ia brown diamond를 $\mathrm{IaA}, \mathrm{IaB}, \mathrm{IaAB}(\mathrm{A}>\mathrm{B}), \mathrm{IaAB}(\mathrm{A}=\mathrm{B}), \mathrm{IaAB}$ 

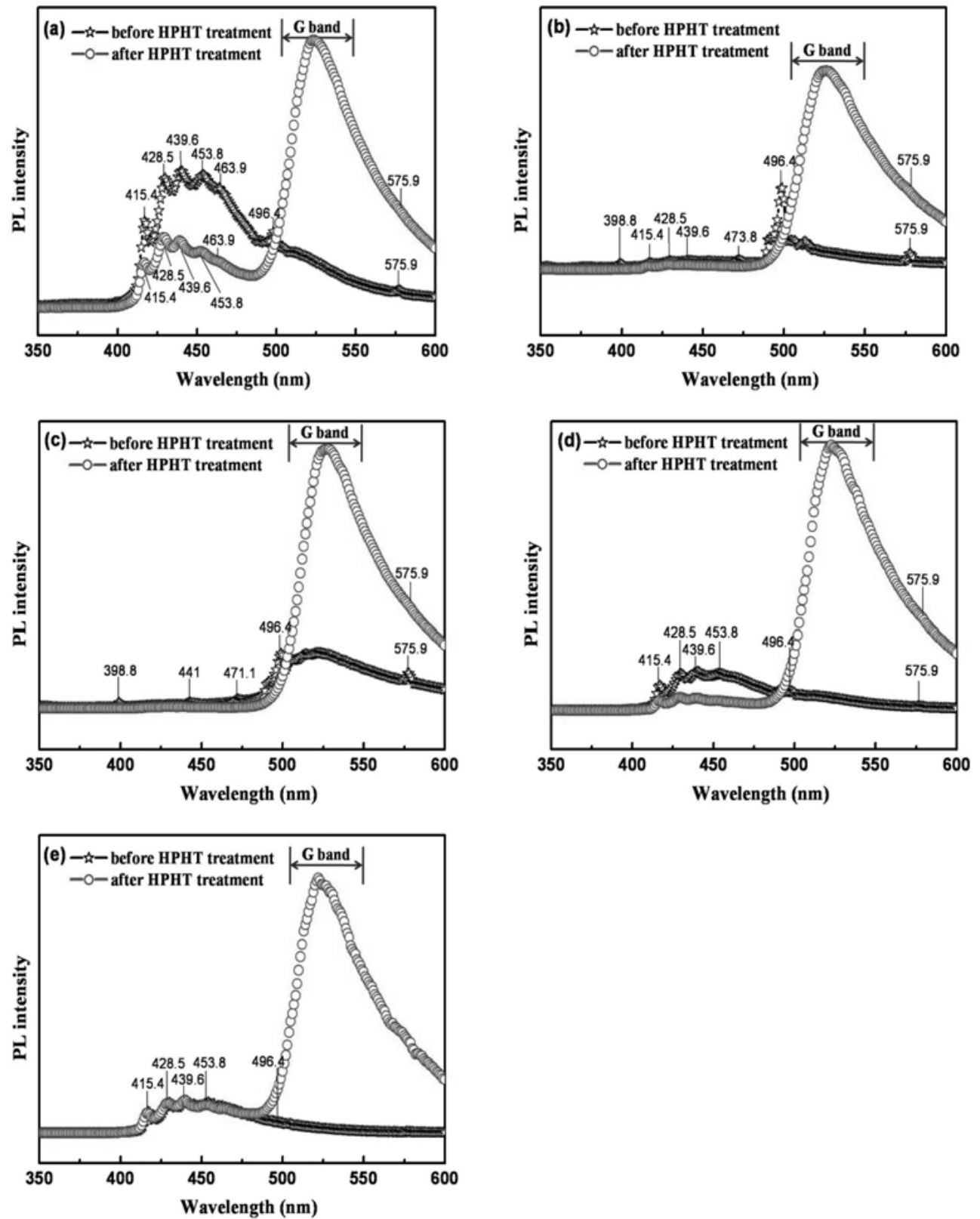

Fig. 8. Photoluminescence peaks of the various types of diamonds before (black line) and after (red line) HPHT treatment using a $325 \mathrm{~nm}$ He-Cd laser source; (a) type IaB, (b) type IaAB (A > B), (c) type IaA, (d) type IaAB $(A=B)$ and (e) type IaAB $(A<B)$.

$(\mathrm{A}<\mathrm{B})$ 로 세부적으로 분류하고, 보석학적 기초검사와 더 불어 다양한 분광학적 분석을 통하여 amber center의 유 무를 확인하였고, HPHT 처리 전과 후 질소결합상태의 변 화 등을 비교 관찰하였다. HPHT처리 후 모든 시료는 N3 center $(415.4 \mathrm{~nm}), \mathrm{H} 4$ center $(496.4 \mathrm{~nm})$ 및 platelet와 연 관된 $\left(1363 \mathrm{~cm}^{-1}\right)$ 의 peak가 감소하였고, $\mathrm{H} 3$ center $(503.2$ $\mathrm{nm}$ )와 G-band가 증가하는 경향을 나타냈다. 그 결과로 인해 HPHT 처리 후 다이아몬드의 color가 brown에서 yellowish green으로 바뀌었고, 다이아몬드 color변화의 원 인은 HPHT처리에 의해 발생된 H3 center, G-band 및
$\mathrm{H} 2$ center의 생성에 기인한 것으로 사료된다. 또한 HPHT 처리 시 질소의 $\mathrm{B}$ 집합보다 $\mathrm{A}$ 집합이 더 감소하는 경향 을 나타내었으며, $\mathrm{A}$ 또는 B집합의 파괴에서 발생된 질소 원자에 의해 질소의 interstitial center $(594 \mathrm{~nm})$ 가 증가함 을 알 수 있었다. HPHT 처리 후 모든 시료는 $(\mathrm{N}-\mathrm{V})^{-}$ center가 생성됨을 확인 할 수 있었다. 결론적으로 HPHT 처리를 통해 다이아몬드 내에 존재하는 질소결합관련 상 태의 변화를 확인할 수 있었고, amber center는 모든 brown 다이아몬드에 나타나는 것이 아님을 확인할 수 있 었다. 비록 본 연구에 사용된 brown 다이아몬드의 경우 

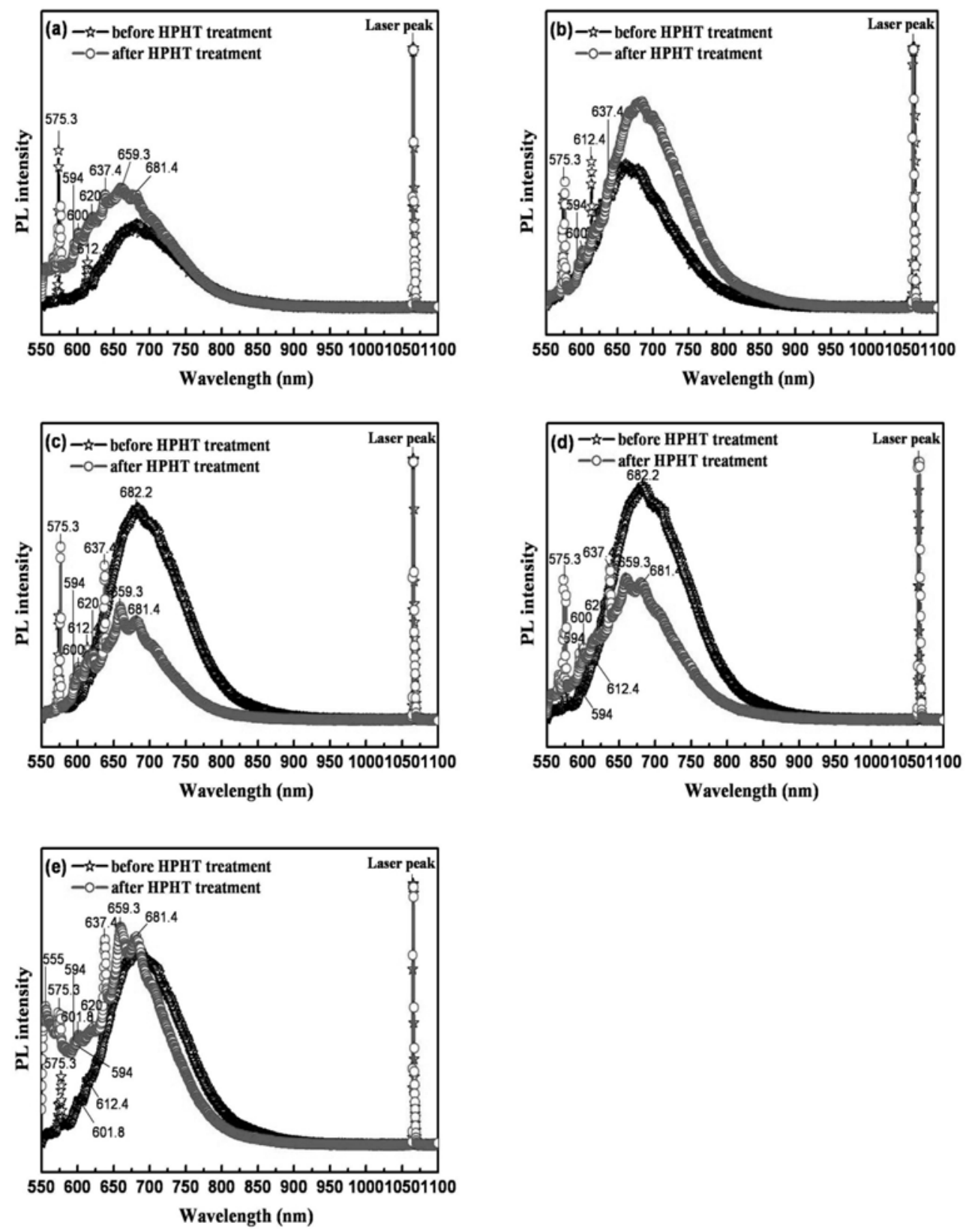

Fig. 9. Photoluminescence peaks of the various types of diamonds before (black line) and after (red line) HPHT treatment using a $532 \mathrm{~nm}$ green laser source; (a) type IaB, (b) type IaAB $(A>B)$, (c) type IaA, (d) type IaAB $(A=B)$ and (e) type IaAB $(A<B)$.

amber center의 부재로 HPHT처리 전후의 amber center 의 변화를 확인하지 못하였지만, amber center와 brown color의 발색 원인에 대한 관련성 조사는 차후에도 계속 수행되어야 할 것이다.

\section{참 고 문 헌}

1. I. Kiflawi, A. Mainwood, H. Kanda and D. Fisher, Phys. Rev. B., 54(23), 16719 (1996).

2. K. Iakoubovskii, I. Kiflawi, K. Johnston, A. T. Collins,
G. Davies and A. Stesmans, Physica B., 340-342, 67 (2003).

3. I. Kiflawi, A. T. Collins, K. Iakoubovskii and D. Fisher, J. Phys. Condens. Matter, 19, 046216 (2007).

4. A. T. Collins, H. Kanda and H. Kitawaki, Diamond Relat. Mater., 9, 113 (2000).

5. V. G. Vins and O. V. Kononov, Diamond Relat. Mater., 12, 542 (2003).

6. A. T. Collins, Diamond Relat. Mater., 12, 1976 (2003).

7. J. E. Shigley and E. Fritsch, J. Gemmol., 23, 259 (1993).

8. F. D. Weert and J. V. Royen, Diamond Relat. Mater., 10, 474 (2001). 
9. F. D. Weerdt and A. T. Collins, Diamond Relat. Mater., 16, 512 (2007).

10. A. T. Collins and K. Mohammed, J. Phys. C. Solid State Phys., 15, 147 (1982).

11. Y. Mita, Y. Yamada, Y. Nisida, M. Okada and T. Nakashima, Physica B, 376-377, 288 (2006).

12. L. Msssi, E. Fritsch, A. T. Collins, T. Hainschwang and F. Notari, Diamond Relat. Mater., 14, 1623 (2005).
13. A. M. Zaitsev, Optical Properties of Diamond : A Data Handbook, p.46-65, Springer-Verlag Heidelberg, New York (2001).

14. A.T. Collins, A. Connor, Ly. Cheng-Han and A. Shareef, J. Applied Phys., 97, 083518 (2005)

15. P. R. Buerki, I. M. Reinitz, S. Muhlmeister and S. Elen, Diamond Relat. Mater., 8, 1061 (1999). 